\title{
A Survey of Normal Form Covers for Context Free Grammars
}

\author{
Anton Nijholt \\ Vrije Universiteit, Dept. of Mathematics, PO-Box 7161, Amsterdam, The Netherlands
}

Summary. An overview is given of cover results for normal forms of contextfree grammars. The emphasis in this paper is on the possibility of constructing $\varepsilon$-free grammars, non-left-recursive grammars and grammars in Greibach normal form. Among others it is proved that any $\varepsilon$-free context-free grammar can be right covered with a context-free grammar in Greibach normal form.

All the cover results concerning the $\varepsilon$-free grammars, the non-left-recursive grammars and the grammars in Greibach normal form are listed, with respect to several types of covers, in a cover-table.

\section{Introduction}

We study the existence and nonexistence of grammar covers for some normal forms for context-free grammars. That is, we consider problems in which we ask: Given classes of grammars $\Gamma_{1}$ and $\Gamma_{2}$, can we find for each grammar $G$ in $\Gamma_{1}$ a grammar $G^{\prime}$ in $\Gamma_{2}$ such that $G^{\prime}$ covers $G$ ?

For $\Gamma_{1}$ we will consider arbitrary context-free grammars. Moreover, by introducing some conditions which should be satisfied we consider also some subclasses of the context-free grammars. For $\Gamma_{2}$ we will concentrate on the $\varepsilon$-free, the non-left-recursive and the Greibach normal form grammars.

A context-free grammar $G^{\prime}$ is said to cover a context-free grammar $G$ if it is possible to define a homomorphism between the parses of $G^{\prime}$ and those of $G$.

We will restrict ourselves to covers which are defined with the help of left and right parses of the grammars in question. It follows that we can define four types of covers, viz. we can define covers in such a way that left parses are mapped on left parses, left parses are mapped on right parses, right parses are mapped on left parses or right parses are mapped on right parses. For each of these covers we will present a yes or no answer to the question whether several types of context-free grammars can be covered by grammars in a certain normal form.

A variety of results in this research area have been obtained before (cf. Aho 
and Ullman [1], Gray and Harrison [6, 7], Nijholt [21, 22, 23, 24], SoisalonSoininen [29] and Ukkonen [31-33 and unpublished]. The aim of this paper is to give a complete overview of the relevant cover results for normal forms of context-free grammars. That is, we collect some of the results in the above mentioned papers and we fill in the missing parts.

The concept of grammar cover can be considered as a grammatical similarity relation. Many other relations between grammars have been defined. For example, there is the concept of structural equivalence (Paull and Unger [27]), there is the grammar functor or $X$-functor approach, initiated by Hotz $[10,11]$, and there are the grammar forms introduced by Cremers and Ginsburg [3].

One motivation to consider these relations can just be the mathematical interest in comparing and relating different subclasses of the context-free grammars. Especially in the case of normal forms of context-free grammars it is natural to ask whether a grammar belonging to a certain class can be transformed to a grammar in a certain normal form and to determine which relations hold between the two grammars. Dependent on this relation one can then conclude that the transformation preserves certain properties of the original grammar.

For each similarity relation there are some obvious questions concerning decidability and complexity. In Hunt, Rosenkrantz and Szymanski [15, 16] decidability results for context-free grammars with respect to the grammar cover are presented. Among others it is shown that it is undecidable whether a context-free grammar $G^{\prime}$ covers a context-free grammar $G$. An overview of complexity results for grammatical similarity relations is given in Hunt and Rosenkrantz [14]. The second motivation to consider grammar covers is their proven usefulness in the theory and practice of parsing and compiler building. Immediately after the presentation of the cover definition we will return to this aspect.

The organization of this paper is as follows. After the presentation of some preliminaries there is a short section in which we discuss the grammar cover concept and how it has appeared, sometimes defined in an informal way, in the literature. In Sect. 2 we list some general theorems on the existence of covering grammars. New theorems and corresponding transformations on context-free grammars to produce grammars in Greibach normal form are also presented in this section.

As the main result of this section we consider that we are able to show that any $\varepsilon$-free context-free grammar can be transformed to a context-free grammar in Greibach normal form in such a way that a right cover (in this case right parses can be mapped on right parses) can be defined.

In Sect. 3 we present an adapted version of a grammar which is due to Ukkonen [33]. Together with the results and observations in Sect. 2 this grammar is sufficient to obtain all negative cover results which are relevant for the classes of grammars which we consider. The example in this section is chosen in such a way that some cover results for strict deterministic, $L L(k)$ and $L R(k)$ grammars become obvious.

Finally, in Sect. 4 a (cover-) table is constructed in which all the results are listed. 
For a survey of normal form cover results for regular grammars the reader is referred to Nijholt [26]. A survey which includes results for $L L(k), L R(k)$ and strict deterministic grammars is in preparation. In Mickunas [19], Mickunas, Lancaster and Schneider [20] and in Nijholt [25] other cover results for $L R(k)$ grammars can be found.

Results for the grammar functor approach for normal forms of context-free grammars can be found in Hotz [12] and Benson [2] and for the $L L(k)$ and $L R(k)$ grammars in Hotz and Ross [13] and in Ross, Hotz and Benson [28].

\subsection{Preliminaries}

We review various commonly known definitions (cf. Aho and Ullman [1]) and give some notations.

A context-free grammar (CFG) will be denoted with the usual fourtuple $G$ $=(N, \Sigma, P, S)$, where $N$ is the set of nonterminal symbols (generally denoted by the Roman capitals $A, B, C, \ldots$ ), $\Sigma$ is the set of terminal symbols (denoted by the smalls $a, b, c \ldots), P \subseteq N \times(\Sigma \cup N)^{*}$ is the set of productions (we use the notation $A \rightarrow \alpha$ if $(A, \alpha) \in P)$ and $S \in N$ is the start symbol. We define $V=N \cup \Sigma$. Elements of $V$ will generally be denoted by $X, Y$ and $Z$; elements of $V^{*}$ by the Greek smalls $\alpha, \beta, \gamma, \ldots$ and elements of $\Sigma^{*}$ by the smalls $u, v, w, x, y$ and $z$. We have the usual notations $\Rightarrow, \stackrel{+}{\Rightarrow}$ and $\stackrel{*}{\Rightarrow}$ for derivations and we use indices $L$ and $R$ to denote leftmost and rightmost derivations, respectively. The language generated by $G$ is the set $L(G)=\left\{w \in \Sigma^{*} \mid S \stackrel{*}{\Rightarrow} w\right\}$.

The sequence of productions which are used in a leftmost derivation from $S$ to a string $w \in \Sigma^{*}$ is called a left parse for $w$. The reverse of a sequence of productions in such a rightmost derivation is called a right parse for $w$.

If $\alpha \in V^{*}$ then $\alpha^{R}$ denotes the reverse of $\alpha$ and $|\alpha|$ denotes the length of $\alpha$. The symbol $\varepsilon$ is reserved for the empty string (the string with length zero). If $|\alpha|<k$ then $k: \alpha$ denotes $\alpha$, otherwise $k: \alpha$ denotes the prefix of $\alpha$ with length $k$.

If $Q$ is a set then $|Q|$ denotes the number of elements in $Q$. For each CFG $G$ $=(N, \Sigma, P, S)$ we define $\Delta_{G}=\{i|1 \leqq i \leqq| P \mid\}$, the set of production numbers of $G$. If $A \rightarrow \alpha$ is the ith production in $P$ then we sometimes write $i \cdot A \rightarrow \alpha$. Moreover, we write $A \stackrel{\pi}{\Rightarrow} \alpha$, where $\pi \in \Delta_{G}^{*}$, if the derivation from $A$ to $\alpha$ is done according to the sequence of productions $\pi$. Hence, if $S \underset{\mathbf{L}}{\stackrel{\pi}{\Longrightarrow}} w$ then $\pi$ is a left parse for $w$ and if $S \underset{R}{\stackrel{\pi}{\Rightarrow}} w$ then $\pi^{R}$ is a right parse for $w$.

The degree of ambiguity of a sentence $w \in L(G)$ is the number of different left parses for $w$. Notation: $\langle w, G\rangle$. If for any $w \in L(G)$ we have $\langle w, G\rangle=1$ then $G$ is called unambiguous.

For any $A \in N$ we define $\operatorname{rhs}(A)=\{\alpha \mid A \rightarrow \alpha$ is in $P\}$.

Definition 1.1. A CFG $G=(N, \Sigma, P, S)$ is said to be

a) $\varepsilon$-free, if $P \subseteq N \times V^{+}$.

b) cycle-free, if for any $A \in N$ a derivation $A \stackrel{+}{\Rightarrow} A$ does not exist. 
c) non-left-recursive (NLR), if for any $A \in N$ and $\alpha \in V^{*}$ a derivation $A \stackrel{+}{\Rightarrow} A \alpha$ does not exist.

d) in Greibach normal form (GNF), if $P \subseteq N \times \Sigma N^{*}$.

We will also use the obvious notion of non-right-recursiveness (NRR) and we use the notation $\overline{\mathrm{GNF}}$ if $P \subseteq N \times N^{*} \Sigma$.

Throughout this paper we assume that the (context-free) grammars in question are cycle-free and that the alphabets of the grammars do not contain useless symbols (cf. Aho and Ullman [1]).

Definition 1.2. a) Let $V_{1}$ and $V_{2}$ be alphabets. A homomorphism is a mapping $\psi: V_{1} \rightarrow V_{2}^{*}$. The domain of the homomorphism $\psi$ is extended to $V_{1}^{*}$ by letting $\psi(\varepsilon)=\varepsilon$ and $\psi(\alpha a)=\psi(\alpha) \psi(a)$ for all $\alpha \in V_{1}^{*}$ and $a \in V_{1}$. We say that $\psi$ is fine if $\psi: V_{1} \rightarrow V_{2} \cup\{\varepsilon\}$ and very fine if $\psi: V_{1} \rightarrow V_{2}$.

b) Let $G=(N, \Sigma, P, S)$ be a CFG. We define $\tau_{l}(G)=\{(w, \pi) \mid S \underset{\sim}{\stackrel{\pi}{\Rightarrow}} w\}$ and $\tau_{\bar{r}}(G)$ $=\left\{\left(w, \pi^{R}\right) \mid S \underset{R}{\stackrel{\pi}{\Rightarrow}} w\right\}$.

c) Assume that $x, y \in\{l, \bar{r}\}$. A CFG $G=(N, \Sigma, P, S)$ is said to $x$-to-y cover a CFG $G^{\prime}=\left(N^{\prime}, \Sigma^{\prime}, P^{\prime}, S^{\prime}\right)$ if there exists a homomorphism $\psi: \Delta_{G^{\prime}} \rightarrow \Delta_{G}^{*}$ such that

(i) if $\left(w, \pi^{\prime}\right) \in \tau_{x}\left(G^{\prime}\right)$ then $\left(w, \psi\left(\pi^{\prime}\right)\right) \in \tau_{y}(G)$, and

(ii) if $(w, \pi) \in \tau_{y}(G)$ then there exists $\pi^{\prime}$ such that $\left(w, \pi^{\prime}\right) \in \tau_{x}\left(G^{\prime}\right)$ and $\psi\left(\pi^{\prime}\right)=\pi$.

Clearly, if $G^{\prime} x$-to-y covers $G$ then $L(G)=L\left(G^{\prime}\right)$ and $\left\langle w, G^{\prime}\right\rangle \geqq\langle w, G\rangle$. To denote that a production $A \rightarrow \alpha$ (or $i \cdot A \rightarrow \alpha$ ) is mapped on a string $\pi$ of productions by a (cover) homomorphism we will sometimes write $A \rightarrow \alpha\langle\pi\rangle$ (or $i \cdot A \rightarrow \alpha\langle\pi\rangle)$.

For the original cover definition the reader is referred to Gray and Harrison $[6,7]^{1}$ (cf. also Aho and Ullman [1]). A more general treatment of covers can be found in Nijholt [24]. The following notation will be useful.

Notation. a) $G^{\prime}[l / l] G$, if $G^{\prime}$ left-to-left covers $G$ (left cover).

b) $G^{\prime}[l / \vec{r}] G$, if $G^{\prime}$ left-to-right covers $G$.

c) $G^{\prime}[\bar{r} / l] G$, if $G^{\prime}$ right-to-left covers $G$.

d) $G^{\prime}[\bar{r} / \bar{r}] G$, if $G^{\prime}$ right-to-right covers $G$ (right cover).

In one of the main transformations of this paper we will use chains and left production chains.

Definition 1.3. Let $G=(N, \Sigma, P, S)$ be a CFG.

a) Define a relation $C H \subseteq V \times N^{*} \Sigma$ as follows. If $X_{0} \in N$ then $C H\left(X_{0}\right)$, the set of chains of $X_{0}$ is defined by

$$
C H\left(X_{0}\right)=\left\{X_{0} X_{1} \ldots X_{n} \mid X_{0} \underset{L}{\Rightarrow} X_{1} \psi_{1} \underset{L}{\Rightarrow} \ldots \underset{L}{\Rightarrow} X_{n} \psi_{n}, \psi_{i} \in V^{*}, 1 \leqq i \leqq n\right\},
$$

and for $c \in \Sigma$,

$$
C H(c)=\{c\}
$$

\footnotetext{
1 It should be observed that our cover definition is slightly different from theirs. Gray and Harrison's definition of complete cover may be compared with our definition of cover if we demand that the homomorphism is fine
} 
b) Define a relation $L P \subseteq N^{*} \Sigma \times \Delta_{G}^{*}$ as follows. Let $\pi=X_{0} X_{1} \ldots X_{n} \in N^{+} \Sigma$, then $L P(\pi)$, the set of left production chains of $\pi$, is defined by

$$
L P(\pi)=\left\{i_{0} i_{1} \ldots i_{n-1} \mid X_{0} \stackrel{i_{0}}{\Rightarrow} X_{1} \psi_{1} \underset{L}{\stackrel{i_{1}}{\Rightarrow}} \ldots \stackrel{i_{n-1}}{\Longrightarrow} X_{n} \psi_{n}, \psi_{i} \in V^{*}, 1 \leqq i \leqq n\right\}
$$

If $\pi \in \Sigma$ then $L P(\pi)=\{\varepsilon\}$.

\subsection{Covers and Parsing}

Let $G=(N, \Sigma, P, S)$ be a CFG. A parser for $G$ determines whether a string $w$ of symbols is in $L(G)$ and if so it produces a parse tree for $w$ with respect to $G$. Either left parses or right parses will be used to represent a parse tree. Once the parse tree is known, code generation can take place. Various persing methods have been introduced for the class of context-free grammars and its subclasses. For each parsing method there is a class of grammars which are suitable for this method. One can try to transform a grammar to make it suitable for a chosen parsing method or to improve its parsing properties. If this transformation can be done in such a way that the new grammar $G^{\prime}$ covers the original grammar $G$, then we can parse with respect to $G^{\prime}$ and, by applying the cover homomorphism, obtain the parse with respect to $G$. This is illustrated in Fig. 1.

It is usual to distinguish between top-down parsing and bottom-up parsing. In top-down parsing the goal is to find a left parse while in bottom-up parsing the goal is a right parse. Both for top-down parsing as for bottom-up parsing there exist conditions which, when satisfied by the grammar, can improve the parsing. A well-known condition for (deterministic) top-down parsing is that the grammar should be non-left-recursive. Grammars in GNF are non-left-recursive.

It has been observed in Griffiths and Petrick [8] that the original GNF transformation distorts the structure of the grammar in such a way that ... "To date, no efficient procedure for relating the structural descriptions of Greibach normal form grammars to the context-free grammars from which they were constructed has been found". Further investigations on this problem can be found in Kuno [17], Kurki-Suoni [18], Foster [4,5] and Stearns [30]. The latter three authors do in fact use, in an informal way, the notion of a right cover. Gray and Harrison [6,7] gave a formal definition of right covers. Their definition, which slightly differs from ours, was inspired by cover definitions

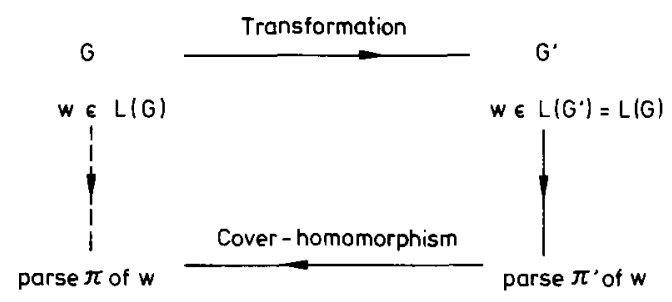

Fig. 1 
which appear in unpublished work of J.C. Reynolds and R. Haskell. SoisalonSoininen [29] has translated the results of Kurki-Suonio in the cover-formalism.

As mentioned in Nijholt [23] there has been some confusion on the possibility to cover grammars with grammars in GNF. In this paper we will give a transformation from arbitrary grammars to grammars in GNF such that a right cover can be defined.

\section{Theorems and Transformations}

This section contains a rather long list of theorems and transformations which are necessary to construct the cover-table which is presented in Sect. 4. For some algorithms and proofs the reader is referred to other papers. None of the algorithms not given here does have a complicated proof of correctness.

\subsection{General Results}

Our first results deal with some general observations on covers for context-free grammars. Firstly we will slightly generalize the cover definition in order to be able to present the following lemma. In the remainder of this paper we will not refer to this lemma if it is used. We will admit covers which are defined with the help of reversed left and right parses. We use $\bar{l}$ and $r$ to denote them. Moreover, for any $x \in\{\bar{l}, \bar{r}\}$ we will write $\bar{x}=x$.

Lemma 2.1. If $G^{\prime}[x / y] G$ then $G^{\prime}[\bar{x} / \bar{y}] G$.

Proof. Suppose that $G^{\prime}[x / y] G$ under a cover-homomorphism $\psi$. Define $\psi^{\prime}(i)$ $=(\psi(i))^{R}$ for any $i \in \Delta_{G^{\prime}}$. Homomorphism $\psi^{\prime}$ is the cover-homomorphism under which $G^{\prime}[\bar{x} / \bar{y}] G$.

Theorem 2.1. a) For any $C F G G$ there exists a $C F G G^{\prime}$ such that $G^{\prime}[l / \bar{r}] G$.

b) For any $C F G G$ there exists a $C F G G^{\prime}$ such that $G^{\prime}[\bar{r} / l] G$.

Proof. (a) Grammar $G^{\prime}$ is constructed from CFG $G$ by defining

$$
P^{\prime}=\left\{A \rightarrow \alpha H_{i}\langle\varepsilon\rangle \mid i \cdot A \rightarrow \alpha \text { is in } P\right\} \cup\left\{H_{i} \rightarrow \varepsilon\langle i\rangle|1 \leqq i \leqq| P \mid\right\} .
$$

The symbols $H_{i}, 1 \leqq i \leqq|P|$ are newly introduced nonterminal symbols which are added to $N$ to obtain $N^{\prime}$.

(b) Grammar $G^{\prime}$ is constructed from CFG $G$ by defining

$$
P^{\prime}=\left\{A \rightarrow H_{i} \alpha\langle\varepsilon\rangle \mid i \cdot A \rightarrow \alpha \text { is in } P\right\} \cup\left\{H_{i} \rightarrow \varepsilon\langle i\rangle|1 \leqq i \leqq| P \mid\right\} .
$$

The symbols $H_{i}, 1 \leqq i \leqq|P|$ are newly introduced nonterminal symbols which are added to $N$ to obtain $N^{\prime}$.

The following observation on 'symmetry' will be very useful if we construct the cover-table in Sect. 4.

Observation 2.1. Let $G=(N, \Sigma, P, S)$ be a CFG. Define $G^{R}=\left(N, \Sigma, P^{R}, S\right)$ by letting $P^{R}=\left\{A \rightarrow \alpha^{R} \mid A \rightarrow \alpha\right.$ is in $\left.P\right\}$. Notice that a leftmost derivation of a 
sentence $w \in L(G)$ coincides with a rightmost derivation of $w^{R} \in L\left(G^{R}\right)$. In what follows we will frequently make use of this 'symmetry'. For example, if a grammar $G$ can not be left covered by an $\varepsilon$-free grammar then it follows (cf. also Lemma 2.1) that $G^{R}$ can not be right covered by an $\varepsilon$-free grammar. Another example is the situation in which a grammar $G$ does not have a left-to-right covering grammar in GNF. Then $G^{R}$ does not have a right-to-left covering grammar in $\overline{\mathrm{GNF}}$.

\subsection{Non-Left-Recursive Grammars}

Next we turn our attention to results which show the possibility of finding nonleft-recursive grammars for 'arbitrary' context-free grammars.

Observation 2.2. If CFG $G$ in Theorem 2.1 is non-left-recursive then (both in a) and b)) $G^{\prime}$ is non-left-recursive.

Any $\varepsilon$-free CFG $G$ (cycle-free, no useless symbols) can be transformed to a NLR grammar $G^{\prime}$ such that $G^{\prime}[\bar{r} / \bar{r}] G$ and $G^{\prime}[l / \bar{r}] G$. This result first appeared in Nijholt [22]. Soisalon-Soininen [29] gave a more simple proof of this result. One of the transformations which is used in the latter paper is based on an idea of Kurki-Suonio [18]. This trick can also be used for a transformation presented in Wood [34] and which is due to J.M. Foster.

Corollary 2.1. Any $\varepsilon$-free $C F G G$ can be transformed to a $C F G G^{\prime}$ such that $G^{\prime}$ is $N L R$ and such that $G^{\prime}[l / \bar{r}] G$ and $G^{\prime}[\bar{r} / \bar{r}] G$.

Each of the above mentioned methods to obtain the NLR grammar $G^{\prime}$ can be adapted in a very simple way in order to obtain an $\varepsilon$-free NLR grammar $G^{\prime \prime}$ such that $G^{\prime \prime}[\bar{r} / \bar{r}] G$. This result can also be obtained from a more general observation of Ukkonen [32, and unpublished] which we give, slightly adapted, below.

Corollary 2.2. Any NLR grammar $G$ can be transformed to an $\varepsilon-$ free NLR grammar $G^{\prime}$ such that $G^{\prime}[\bar{r} / \bar{r}] G .^{2}$

In Ukkonen's algorithm for eliminating $\varepsilon$-productions from a grammar $G$ $=(N, \Sigma, P, S)$ it is assumed that if $\varepsilon \in L(G)$ then there do not exist two different rightmost derivations to $\varepsilon$. Since in our definition of $\varepsilon$-free grammar we have $P \subseteq N \times V^{+}$we do not bother about introducing a special production $S^{\prime} \rightarrow \varepsilon$ for grammar $G^{\prime}$. Hence, in Corollary 2.2 we have $L\left(G^{\prime}\right)=L(G) /\{\varepsilon\}$.

The following corollary follows from the transitivity of the cover relation.

Corollary 2.3. Any $\varepsilon$-free $C F G G$ can be transformed to an $\varepsilon$-free $N L R$ grammar $G^{\prime}$ such that $G^{\prime}[\bar{r} / \bar{r}] G$.

With this corollary we conclude our observations on finding non-leftrecursive grammars.

2 It is assumed that if $\varepsilon \in L(G)$ then there do not exist two different rightmost derivations to $\varepsilon$ 


\subsection{Elimination of Single Productions}

Before we turn our attention to the problem of finding grammars in GNF we have a few remarks on some special conditions. Consider a CFG $G$ with productions $S \rightarrow A, S \rightarrow B, A \rightarrow a$ and $B \rightarrow a$. Suppose we want to find an equivalent $\varepsilon$-free grammar without single productions (i.e. productions of the form $X \rightarrow Y$ with both $X$ and $Y$ in $N$ ). There is only one grammar which has this property, grammar $G^{\prime}$ with the one production $S^{\prime} \rightarrow a$. It follows that in genral elimination of single productions can not be done in such a way that a left or right cover can be defined since condition (ii) of the cover definition can not always be satisfied.

In some cases we find it convenient to talk about grammars without single productions. Although it is not always necessary (in some cases we could use more refined conditions) we assume for a few algorithms in the remainder of this paper that they have an input grammar without single productions. We use a rather rude approach to solve the problem of eliminating single productions. The method which is in the proof of the following theorem was first shown in [21] and we include it here. It should be observed that a more simple method can be used if we allow, as is possible in the grammar functor approach, that one production can have different labels. However, from the point of view of parsing we recognize productions rather than labels. Therefore we use the following method.

Theorem 2.2. Let $G=(N, \Sigma, P, S)$ be an e-free $C F G$. Grammar $G_{0}=\left(N \cup\left\{S_{0}\right\}\right.$, $\Sigma \cup\{\perp\}, P \cup\left\{S_{0} \rightarrow S \perp\right\}, S_{0}$ ) can be transformed to a $C F G G^{\prime}$ without single productions in such a way that $G^{\prime}[\bar{r} / \bar{r}] G_{0}$ and $G^{\prime}[l / l] G_{0}$.

Proof. We show how the elimination can be done. We use auxiliary sets $P_{0}$ and $P_{1}$. The set $P_{0}$ is the set of all the single productions in $P$. Initially $P_{1}$ $=\left\{A \rightarrow \alpha\langle i\rangle \mid i \cdot A \rightarrow \alpha\right.$ is in $\left.P-P_{0}\right\}, N^{\prime}=N$ and $P^{\prime}=\emptyset$.

(i) Let $A \in N$. If $A \stackrel{\delta}{\Rightarrow} \beta \stackrel{i}{\Rightarrow} \gamma$ is a derivation in $G$ such that $\delta \neq \varepsilon$ and either $|\gamma| \geqq 2$ or $\gamma \in \Sigma$ then add $[A \delta i] \rightarrow \gamma\langle\pi\rangle$ to $P_{1}$ and $[A \delta i]$ to $N^{\prime}$. To obtain a left cover define $\pi=\delta i$. To obtain a right cover define $\pi=i \delta^{R}$. Notice that since $G$ is cycle-free there are finitely many derivations to consider.

(ii) Define a homomorphism $h: N^{\prime} \cup \Sigma \rightarrow N \cup \Sigma$ by defining $h(X)=X$ for each $X \in N \cup \Sigma$ and $h([A \pi])=A$ for each $[A \pi] \in N^{\prime}-N$. For each production $A^{\prime} \rightarrow \gamma\langle\pi\rangle$ in $P_{1}$ (hence, $A^{\prime} \in N^{\prime}$ and $\gamma \in V V^{+}$) add the productions in the set $\left\{A^{\prime} \rightarrow \gamma^{\prime}\langle\pi\rangle \mid A^{\prime} \rightarrow \gamma\langle\pi\rangle\right.$ in $P_{1}$ and $\left.h\left(\gamma^{\prime}\right)=\gamma\right\}$ to $P^{\prime}$.

(iii) Remove the useless symbols.

Clearly, grammar $G^{\prime}=\left(N^{\prime}, \Sigma, P^{\prime}, S_{0}\right)$ which is obtained does not have single productions. Grammar $G^{\prime}$ left covers grammar $G$. This follows from the following observations. They can be formally proved by induction on the lengths of the derivations. Similar observations hold for the right cover.

a) If $A \underset{L}{\stackrel{\pi^{\prime}}{\Rightarrow}} w$ in $G^{\prime}$ then $A \underset{L}{\stackrel{\pi}{\Rightarrow}} w$ in $G$, with $\psi\left(\pi^{\prime}\right)=\pi$.

b) If $[A \delta] \underset{L}{\stackrel{\pi^{\prime}}{\Rightarrow}} w$ in $G^{\prime}$ then $A \underset{L}{\stackrel{\pi}{\Rightarrow}} w$ in $G$, with $\psi\left(\pi^{\prime}\right)=\pi$.

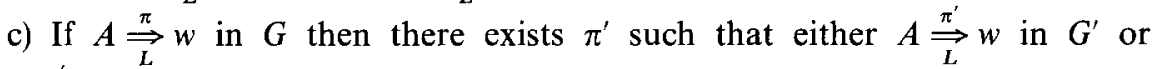
$[A \delta] \underset{L}{\stackrel{\pi^{\prime}}{\Rightarrow}} w$ in $G^{\prime}$, for some $\delta \in \Delta_{G}^{*}$, and with $\psi\left(\pi^{\prime}\right)=\pi$. 
In observation c) we have for $G^{\prime}$ the grammar which is obtained from step (i) and (ii). The implicitly defined cover homomorphism is denoted by $\psi$. This concludes the proof of the theorem.

We emphasize that it is not always necessary to introduce the special production $S_{0} \rightarrow S \perp$. For example, if $G$ is unambiguous. In this case the method mentioned in the proof can be simplified. In fact, only in the case that there exist, for some $a \in \Sigma$, different derivations from $S$ to $a$ it is necessary to introduce this production.

In what follows we do not bother about this special production. The result mentioned in the following observation is an immediate consequence of the method which is used in the proof of Theorem 2.2.

Observation 2.3. If CFG $G$ in Theorem 2.2 is non-left-recursive then CFG $G^{\prime}$ without single productions is also non-left-recursive.

\subsection{Grammars in Greibach Normal Form}

Now we are sufficiently prepared to consider grammars in GNF. This normal form can be obtained in such a way, from $\varepsilon$-free and non-left-recursive grammars, that a left cover can be defined. This was shown in Nijholt [21]. Moreover, this result is a special case of a more general theorem in Nijholt [24]. In the latter paper a transformation (the 'left part transformation') is used which we will recall here. This transformation will be used later, in an adapted form, to obtain right cover results.

We use a special alphabet which is defined below.

Definition 2.1. Let $G=(N, \Sigma, P, S)$ be a CFG. Define the set

$$
[N]=\left\{[A i \alpha] \mid i \cdot A \rightarrow \alpha \beta \text { is in } P, \beta \in V^{*}\right\}
$$

and a homomorphism $\xi:[N] \rightarrow[N]$ by letting $\xi([A i \alpha])$ is

(i) $\varepsilon$ if $i \cdot A \rightarrow \alpha$ is in $P$.

(ii) $[A i \alpha]$ if $i \cdot A \rightarrow \alpha \beta$ is in $P$, with $\beta \neq \varepsilon$.

We present the algorithm in such a way that each newly obtained production is followed by its image under a cover-homomorphism $\psi$ for a left cover.

Algorithm 2.1. Input. An \&-free $N L R$ grammar $G=(N, \Sigma, P, S)$ without single productions. Qutput. A CFG $G^{\prime}=\left(N^{\prime}, \Sigma, P^{\prime},[S]\right)$ in $G N F$ such that $G^{\prime}[l / l] G$. Method. The set $P^{\prime}$ consists of all the productions which are introduced below. Set $N^{\prime}$ will contain $[S]$ and all symbols of $[N]$ which appear in the productions. Initially set $\boldsymbol{P}^{\prime}=\emptyset$.

(i) For each pair $(\pi, \rho), \pi=S X_{1} X_{2} \ldots X_{n} \in C H(S)$ and $\rho=i_{0} i_{1} \ldots i_{n-1} \in L P(\pi)$, add $[S] \rightarrow X_{n} \xi\left(\left[X_{n-1} i_{n-1} X_{n}\right] \ldots\left[S i_{0} X_{1}\right]\right)\langle\rho\rangle$ to $P^{\prime}$.

(ii) Let $i \cdot A \rightarrow \alpha X_{0} \varphi$ be in $P, \alpha \neq \varepsilon$. For each pair $(\pi, \rho), \pi=X_{0} X_{1} \ldots$ $X_{n} \in C H\left(X_{0}\right)$ and $\rho=i_{0} i_{1} \ldots i_{n-1} \in L P(\pi)$, add $[A i \alpha] \rightarrow X_{n} \xi\left(\left[X_{n-1} i_{n-1} X_{n}\right] \ldots\right.$ $\left.\left[X_{0} i_{0} X_{1}\right]\left[A i \alpha X_{0}\right]\right)\langle\rho\rangle$ to $P^{\prime}$. 
Notice that for this algorithm the condition that the input grammar $G$ does not have single productions is not a necessary condition. It would be sufficient to demand that, for any $A \in N$ and $X \in V$, if $A \stackrel{\pi}{\Rightarrow} X$ and $A \stackrel{\pi}{\Rightarrow} X$ then $\pi=\pi^{\prime}$. However, as we have shown the single productions can be eliminated in a simple way and we can avoid the introduction of new conditions.

Theorem 2.3. Each 8-free NLR grammar $G$ can be transformed to a $C F G G^{\prime}$ in $G N F$ such that $G^{\prime}[l / l] G$.

Proof. We assume that the single productions have been eliminated. We use Algorithm 2.1 to transform $G$ to a grammar $G^{\prime}$. Clearly, $G^{\prime}$ is in GNF. The cover homomorphism which is implicitly defined in the algorithm is denoted by $\psi$. We use two claims.

Claim 1. If $[A i \alpha] \underset{L}{\stackrel{\pi^{\prime}}{\Rightarrow}} w$ in $G^{\prime}$ then there exists $i \cdot A \rightarrow \alpha \varphi$ in $P, \varphi \neq \varepsilon$, such that $\varphi \underset{L}{\stackrel{\pi}{\Rightarrow}} w$ in $G$, with $\pi=\psi\left(\pi^{\prime}\right)$.

Proof of Claim 1. Induction on $\left|\pi^{\prime}\right|$.

Basis. Assume $\left|\pi^{\prime}\right|=1$. In this case we have a production $\pi^{\prime} \cdot[A i \alpha] \rightarrow w$ in $P^{\prime}$ with $w \in \Sigma$. This production is obtained from either a production $i \cdot A \rightarrow \alpha w$ in $P$ such that $\pi=\varepsilon$ and $\psi\left(\pi^{\prime}\right)=\varepsilon$ or from productions $i \cdot A \rightarrow \alpha X_{0}$ and $j \cdot X_{0} \rightarrow w$, with $\psi\left(\pi^{\prime}\right)$ $=j$.

Induction. Assume $\left|\pi^{\prime}\right|>1$. We can write

$$
[A i \alpha] \stackrel{i^{\prime}}{\longrightarrow} a \xi\left(\left[X_{n-1} i_{n-1} X_{n}\right] \ldots\left[X_{0} i_{0} X_{1}\right]\left[A i \alpha X_{0}\right]\right) \stackrel{\pi^{\prime \prime}}{\longrightarrow} a v,
$$

where $X_{n}=a, i^{\prime} \pi^{\prime \prime}=\pi^{\prime}$ and $a v=w$.

We can factorize $\pi^{\prime \prime}$ such that

(a) $\pi^{\prime \prime}=\pi_{n}^{\prime} \ldots \pi_{0}^{\prime}, v=w_{n} w_{n-1} \ldots w_{0}$.

(b) If $\xi\left(\left[A i \alpha X_{0}\right]\right) \neq \varepsilon$ then

$$
\left[A i \alpha X_{0}\right] \stackrel{\pi_{0}^{\prime}}{\rightleftharpoons} w_{0}
$$

and it follows from the induction hypothesis that there exists $i \cdot A \rightarrow \alpha X_{0} \varphi_{0}$ such that $\varphi_{0} \underset{L}{\stackrel{\pi_{0}}{\longrightarrow}} w_{0}$, with $\psi\left(\pi_{0}^{\prime}\right)=\pi_{0}$. If $\xi\left(\left[A i \alpha X_{0}\right]\right)=\varepsilon$ then $\varphi_{0}=\pi_{0}=w_{0}=\varepsilon$.

(c) If $\xi\left(\left[X_{k-1} i_{k-1} X_{k}\right]\right) \neq \varepsilon, 0<k \leqq n$, then

$$
\left[X_{k-1} i_{k-1} X_{k}\right] \stackrel{\pi_{k}^{\prime}}{\longrightarrow} w_{k}
$$

and it follows that there exists $i_{k-1} \cdot X_{k-1} \rightarrow X_{k} \varphi_{k}$ such that $\varphi_{k} \stackrel{\pi_{k}}{\Longrightarrow} w_{k}$, with $\psi\left(\pi_{k}^{\prime}\right)$ $=\pi_{k}$. If $\xi\left(\left[X_{k-1} i_{k-1} X_{k}\right]\right)=\varepsilon$ then $\varphi_{k}=\pi_{k}=w_{k}=\varepsilon$. Since $G$ has no single productions, this case can only occur if $k=n$.

Hence, since $\psi\left(i^{\prime}\right)=i_{0} i_{1} \ldots i_{n-1}=\rho$ we obtain a derivation

$$
\varphi=X_{0} \varphi_{0} \stackrel{\rho}{\underset{L}{\longrightarrow}} X_{n} \varphi_{n} \varphi_{n-1} \ldots \varphi_{1} \varphi_{0} \underset{\mathrm{L}}{\stackrel{\delta}{\longrightarrow}} X_{n} w_{n} w_{n-1} \ldots w_{1} w_{0}=a v,
$$

with $\delta=\pi_{n} \ldots \pi_{1} \pi_{0}$ and $\psi\left(\pi^{\prime}\right)=\rho \delta=\pi$. This concludes the proof of Claim 1 . 
If $[S] \underset{L}{\stackrel{\pi^{\prime}}{\longrightarrow}} w$ in $G^{\prime}$ then we can, as we did in the induction part of the proof of Claim 1, distinguish the first production which is used and then factorize $\pi^{\prime}$ in a similar way to obtain the conclusion that $S \underset{L}{\stackrel{\pi}{\vec{L}}} w$ in $G$, with $\psi\left(\pi^{\prime}\right)=\pi$.

Claim 2. Assume $i \cdot A \rightarrow \alpha X_{0} \varphi_{0} \in P, \alpha \neq \varepsilon$. If $X_{0} \varphi_{0} \stackrel{\pi}{\vec{L}} w$ in $G$ then there exists

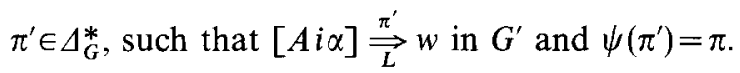

Proof of Claim 2. The proof is by induction on $|\pi|$.

Basis. If $|\pi|=0$ then $X_{0} \varphi_{0} \in \Sigma^{+}$and from the construction it follows that there exists a derivation $[A i \alpha] \underset{\vec{L}}{\stackrel{\pi^{\prime}}{\Rightarrow}} w$ in $G^{\prime}$ such that $\psi\left(\pi^{\prime}\right)=\pi=\varepsilon$. If $|\pi|=1$, then $X_{0} \varphi_{0}$ can be written as $v_{1} B v_{3}$ with $B \in N, v_{1} v_{3} \in \Sigma^{*}$ and $\pi \cdot B \rightarrow v_{2}$ in $P$ with $v_{2} \in \Sigma^{+}$. In this case there exists a derivation

$$
[A i \alpha] \underset{L}{\stackrel{\pi_{1}}{\longrightarrow}} v_{1}\left[A i \alpha v_{1}\right] \stackrel{\pi_{2}}{\underset{L}{\longrightarrow}} v_{1} v_{2} \xi\left(\left[A i \alpha v_{1} B\right]\right) \stackrel{\pi_{3}}{\underset{L}{\longrightarrow}} v_{1} v_{2} v_{3}=w
$$

in $G^{\prime}$ such that $\psi\left(\pi_{1}\right)=\psi\left(\pi_{3}\right)=\varepsilon$ and $\psi\left(\pi_{2}\right)=\pi$.

Induction. Assume $|\pi|>1$. Then there exists $\rho=i_{0} i_{1} \ldots i_{n-1} \in \Delta_{G}^{*}$ such that $i_{k} \cdot X_{k} \rightarrow X_{k+1} \varphi_{k+1}, 0 \leqq k \leqq n-1$, with $X_{n} \in \Sigma$ and such that $\varphi_{k} \underset{L}{\stackrel{\pi_{k}}{\longrightarrow}} w_{k}$. Hence, we can write

$$
X_{0} \varphi_{0} \stackrel{\rho}{\vec{L}} X_{n} \varphi_{n} \ldots \varphi_{1} \varphi_{0} \stackrel{\stackrel{\delta}{\vec{L}}}{w},
$$

with $\delta=\pi_{n} \ldots \pi_{1} \pi_{0}$ and $w=X_{n} w_{n} \ldots w_{1} w_{0}$.

From the construction and the induction hypothesis it follows that (a) $i^{\prime} \cdot[A i \alpha] \rightarrow X_{n} \xi\left(\left[X_{n-1} i_{n-1} X_{n}\right] \ldots\left[A i \alpha X_{0}\right]\right)\langle\rho\rangle$ is in $P^{\prime}$.

(b) If $\varphi_{k} \neq \varepsilon, 0<k \leqq n$, then $\left[X_{k-1} i_{k-1} X_{k}\right] \stackrel{\pi_{k}^{\prime}}{\longrightarrow} w_{k}$, with $\psi\left(\pi_{k}^{\prime}\right)=\pi_{k}$. If $\varphi_{k}=\varepsilon$ then $\pi_{k}=\pi_{k}^{\prime}=w_{k}=\varepsilon$. Since $G$ has no single productions this latter case can only occur if $k=n$.

(c) If $\varphi_{0} \neq \varepsilon$ then $\left[A i \alpha X_{0}\right] \stackrel{\underset{L}{\underline{L}}}{\Rightarrow} w_{0}$, with $\psi\left(\pi_{0}^{\prime}\right)=\pi_{0}$. If $\varphi_{0}=\varepsilon$ then $\pi_{0}=\pi_{0}^{\prime}=w_{0}$ $=\varepsilon$.

Hence, there exists a derivation

$$
[A i \alpha] \underset{\vec{L}}{\stackrel{\pi^{\prime}}{\longrightarrow}} w, \quad \text { with } \pi^{\prime}=i^{\prime} \pi_{n}^{\prime} \ldots \pi_{0}^{\prime} \quad \text { and } \quad \psi\left(\pi^{\prime}\right)=\rho \delta=\pi .
$$

This concludes the proof of Claim 2.

It remains to show that if $S \underset{\vec{L}}{\stackrel{\pi}{\longrightarrow}} w$ in $G$, then $[S] \underset{\vec{L}}{\stackrel{\pi^{\prime}}{\vec{x}}} w$ in $G^{\prime}$, with $\psi\left(\pi^{\prime}\right)=\pi$. However, also in this case this can be shown by proceeding in a way similar to the induction part of the proof of Claim 2. It follows that $G^{\prime}[l / l] G$.

Next we consider the possibility to obtain a CFG in GNF which right covers the $\varepsilon$-free NLR grammar. We use two transformations. Firstly, we transform $\varepsilon$ - 
free NLR grammars to grammars which are almost GNF. For convenience of description we assume that the input grammar is such that terminal symbols in the righthand sides of the productions can only appear at the leftmost positions of the righthand sides. This can be done without loss of generality. For example, if a grammar has a production $i \cdot A \rightarrow \alpha a \beta$, with $\alpha \neq \varepsilon$, then we can replace this production by $A \rightarrow \alpha H_{a} \beta\langle i\rangle$ and $H_{a} \rightarrow a\langle\varepsilon\rangle$ and the new grammar right covers the original grammar. The second transformation will produce GNF grammars from almost GNF grammars.

Definition 2.2. A CFG $G=(N, \Sigma, P, S)$ is said to be an almost GNF grammar if for any production $A \rightarrow \alpha$ in $P$ either

(i) $\alpha \in \Sigma$, or

(ii) $\alpha \in N N^{+}$and $r h s(1: \alpha) \subseteq \Sigma$.

Algorithm 2.2. Input. $A$ NLR grammar $G=(N, \Sigma, P, S)$ such that $P \subseteq N$ $\times\left(\Sigma N^{*} \cup N N^{+}\right)$. Output. An almost $G N F$ grammar $G^{\prime}=\left(N^{\prime}, \Sigma, P^{\prime},[S]\right), G^{\prime}[\bar{r} / \bar{r}] G$. Method. The set $P^{\prime}$ will contain all productions introduced below. The set $N^{\prime}$ will contain $[S]$, all symbols of $[N]$ which appear in the productions and some special indexed symbols $H$. Initially set $P^{\prime}=\emptyset$. to $P^{\prime}$.

(i) For each production of the form $i \cdot S \rightarrow a$ in $P$ with $a \in \Sigma$, add $[S] \rightarrow a\langle i\rangle$

(ii) For each pair $(\pi, \rho), \pi=S X_{1} \ldots X_{n} \in C H(S)$ and $\rho=i_{0} i_{1} \ldots i_{n-1} \in L P(\pi)$, $n>1$, add

and

$$
[S] \rightarrow H_{i_{n-1}} \xi\left(\left[X_{n-1} i_{n-1} X_{n}\right] \ldots\left[S i_{0} X_{1}\right]\right)\langle\varepsilon\rangle
$$

$$
H_{i_{n-1}} \rightarrow X_{n}\langle p\rangle
$$

to $P^{\prime}$. Here, $p=i_{n-1}$ if $i_{n-1} \cdot X_{n-1} \rightarrow X_{n} \in P$ and $p=\varepsilon$ otherwise.

(iii) Let $i \cdot A \rightarrow \alpha X_{0} \varphi$ be in $P, \alpha \neq \varepsilon$. For each pair $(\pi, \rho), \pi$ $=X_{0} X_{1} \ldots X_{n} \in C H\left(X_{0}\right)$ and $\rho=i_{0} i_{1} \ldots i_{n-1} \in L P(\pi)$, the following two cases are distinguished:

(1) $n=1, \varphi=\varepsilon$, and $\xi\left(\left[X_{0} i_{0} X_{1}\right]\right)=\varepsilon ;$ add $[A i \alpha] \rightarrow X_{n}\left\langle i_{0} i\right\rangle$ to $P^{\prime}$.

(2) otherwise, add

and

$$
[A i \alpha] \rightarrow H_{i_{n-1}} \xi\left(\left[X_{n-1} i_{n-1} X_{n}\right] \ldots\left[X_{0} i_{0} X_{1}\right]\left[A i \alpha X_{0}\right]\right)\langle p\rangle
$$

$$
H_{i_{n-1}} \rightarrow X_{n}\langle q\rangle
$$

to $P^{\prime}$, where $p=i$ if $i \cdot A \rightarrow \alpha X_{0}$ is in $P$ and $p=\varepsilon$ otherwise, and $q=i_{n-1}$ if $i_{n-1} \cdot X_{n-1} \rightarrow X_{n} \in P$ and $q=\varepsilon$ otherwise.

Lemma 2.2. Any $\varepsilon$-free $N L R$ grammar $G$ can be transformed to an almost GNF grammar $G^{\prime}$ such that $G^{\prime}[\bar{r} / \bar{r}] G$.

Proof. Without loss of generality we may assume that $G$ does not have single productions. We use Algorithm 2.2 to transform $G$ to a grammar $G^{\prime}$. By construction $G^{\prime}$ is almost GNF.

Claim 1. Cover homomorphism $\psi$, implicitly defined in the algorithm, is welldefined. 
Proof of Claim 1. To verify that for any pair $p$ and $p^{\prime}$ of productions in $P^{\prime}$ it follows that if $\psi(p)=\pi$ and $\psi\left(p^{\prime}\right)=\pi^{\prime}$, with $\pi \neq \pi^{\prime}$ then $p \neq p^{\prime}$. This is straightforward to verify and therefore it is omitted.

In the following claims $\varphi: \Delta_{G^{\prime}} \rightarrow \Delta_{G}^{*}$ is defined by letting, for any $p \in \Delta_{G^{\prime}}, \varphi(p)$ $=\pi^{R}$ iff $\psi(p)=\pi$.

Claim 2. If $[A i \alpha] \stackrel{\pi^{\prime}}{\underset{R}{\longrightarrow}} w$ then $A \stackrel{\varphi\left(\pi^{\prime}\right)}{\longrightarrow} \alpha w$.

Proof of Claim 2. The proof is by induction on $\left|\pi^{\prime}\right|$.

Basis. If $\left|\pi^{\prime}\right|=1$ then $\pi^{\prime}=[A i \alpha] \rightarrow a\langle j i\rangle$. In this case there is a derivation

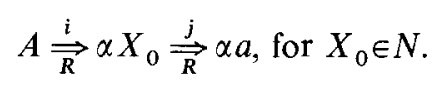

Induction. Assume $\left|\pi^{\prime}\right|=m, m>1$ and assume the property holds for all rightmost derivations with lengths less than $m$. Let

$$
p^{\prime} \cdot[A i \alpha] \rightarrow H_{i_{n-1}} \xi\left(\left[X_{n-1} i_{n-1} X_{n}\right] \ldots\left[X_{0} i_{0} X_{1}\right]\left[A i \alpha X_{0}\right]\right)
$$

be the first production which is used in the derivation $[$ Ai $\alpha] \underset{\boldsymbol{R}}{\stackrel{\pi^{\prime}}{\longrightarrow}} w$. Hence, we may write $w=X_{n} x$ and $\pi^{\prime}=p^{\prime} \gamma q^{\prime}$, where $q^{\prime}=H_{i_{n-1}} \rightarrow X_{n}$. Then we have

$$
\begin{array}{r}
{[A i \alpha] \stackrel{\vec{p}}{\underset{R}{\longrightarrow}} H_{i_{n-1}} \xi\left(\left[X_{n-1} i_{n-1} X_{n}\right] \ldots\left[X_{0} i_{0} X_{1}\right]\left[A i \alpha X_{0}\right]\right) \stackrel{\gamma}{\stackrel{\gamma}{\longrightarrow}} \ldots} \\
\quad \ldots \stackrel{\gamma}{\underset{R}{\longrightarrow}} H_{i_{n-1}} x_{n} x_{n-1} \ldots x_{1} x_{0} \stackrel{q^{\prime}}{\longrightarrow} X_{n} x_{n} x_{n-1} \ldots x_{1} x_{0}=w,
\end{array}
$$

such that

(a) if $\xi\left(\left[A i \alpha X_{0}\right]\right) \neq \varepsilon$ then $\left[A i \alpha X_{0}\right] \underset{R}{\stackrel{\pi_{0}}{\longrightarrow}} x_{0}$, otherwise $x_{0}=\pi_{0}=\varepsilon$,

(b) $\left[X_{k-1} i_{k-1} X_{k}\right] \stackrel{\pi_{k}}{\longrightarrow} x_{k}, 1 \leqq k \leqq n-1$,

(c) if $\xi\left(\left[X_{n-1} i_{n-1} X_{n}\right]\right) \neq \varepsilon$ then $\left[X_{n-1} i_{n-1} X_{n}\right] \underset{R}{\stackrel{\pi_{n}}{\longrightarrow}} x_{n}$, otherwise $\pi_{n}=x_{n}=\varepsilon$, and

(d) $q^{\prime} \cdot H_{i_{n-1}} \rightarrow X_{n}$ with $p^{\prime} \pi_{0} \pi_{1} \ldots \pi_{n} q^{\prime}=p^{\prime} \gamma q^{\prime}=\pi^{\prime}$.

It follows from the induction hypothesis that

(a) $A \stackrel{\varphi\left(p^{\prime} \pi_{0}\right)}{\longrightarrow} \alpha X_{0} x_{0}$, with either $\varphi\left(p^{\prime}\right)=\varepsilon$ or $\pi_{0}=x_{0}=\varepsilon$,

(b) $X_{k-1} \stackrel{\varphi\left(\pi_{k}\right)}{\longrightarrow} X_{k} x_{k}, 1 \leqq k \leqq n-1$, and

(c) $X_{n-1} \stackrel{\varphi\left(\pi_{n} q^{\prime}\right)}{\longrightarrow} X_{n} x_{n}$, with either $\varphi\left(q^{\prime}\right)=\varepsilon$ or $\pi_{n}=x_{n}=\varepsilon$. Thus, $A \stackrel{\varphi\left(\pi^{\prime}\right)}{\longrightarrow} \alpha w$.

Claim 3. Assume that $i \cdot A \rightarrow \alpha X_{0} \varphi$ is in $P$ and $A \underset{R}{\stackrel{i \pi}{\longrightarrow}} \alpha w$. Then there exists $\pi^{\prime} \in \Delta_{G^{\prime}}^{*}$ such that $[A i \alpha] \stackrel{\pi^{\prime}}{=} w$ and $\varphi\left(\pi^{\prime}\right)=i \pi$.

Proof of Claim 3. The proof is by induction on $|\pi|$. 
Basis. If $|\pi|=1$ then, with $\pi \cdot X_{0} \rightarrow w$ in $P, w \in \Sigma$, we have

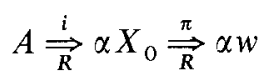

in $G$, and by construction of $G^{\prime}$

$$
[A i \alpha] \stackrel{i^{\prime}}{\Rightarrow} w
$$

with $\varphi\left(i^{\prime}\right)=i \pi$.

Induction. Assume $|\pi|>1$. We factorize

into

$$
A \stackrel{i}{\underset{R}{\longrightarrow}} \alpha X_{0} \varphi \stackrel{\pi}{\stackrel{\pi}{\Rightarrow}} \alpha w
$$

$$
\begin{gathered}
A \stackrel{i}{\stackrel{\boldsymbol{R}}{\rightleftharpoons}} \alpha X_{O} \varphi, \\
\varphi \stackrel{\rho_{1}}{\rightleftharpoons} v_{1}, \quad \text { and } \quad X_{0} \stackrel{\stackrel{\rho_{0}}{\longrightarrow}}{\longrightarrow} a v_{0},
\end{gathered}
$$

where $a v_{0} v_{1}=w$. Since $X_{0} \in N$ we have $\left|\rho_{1}\right|<|\pi|$ and from the induction hypothesis we obtain, if $\varphi \neq \varepsilon$,

$$
\left[A i \alpha X_{0}\right] \stackrel{\stackrel{\rho_{1}^{\prime}}{\longrightarrow}}{\longrightarrow} v_{1}, \quad \text { with } \varphi\left(\rho_{1}^{\prime}\right)=i \rho_{1} .
$$

Moreover, there exist productions $i_{k} \cdot X_{k} \rightarrow X_{k+1} \varphi_{k}, 0 \leqq k \leqq n-1$ and $X_{n}=a$, such that

(i) $X_{k} \stackrel{i_{k}}{\longrightarrow} X_{k+1} \varphi_{k} \underset{R}{\stackrel{\pi_{k}}{\longrightarrow}} X_{k+1} w_{k}$, with $0 \leqq k \leqq n-1$ and such that $\left|\pi_{k}\right|<|\pi|$, hence

$$
\left[X_{k} i_{k} X_{k+1}\right] \underset{R}{\stackrel{\pi_{k}^{\prime}}{\rightleftharpoons}} w_{k} \text { and } \varphi\left(\pi_{k}^{\prime}\right)=i_{k} \pi_{k}
$$

(ii) $X_{n-1} \stackrel{i_{n-1}}{\underset{R}{\longrightarrow}} a \varphi_{n} \stackrel{\pi_{n-1}}{\longrightarrow} a w_{n-1}$, such that $\left|\pi_{n-1}\right|<|\pi|$, hence, if $\varphi_{n} \neq \varepsilon$,

$$
\left[X_{n-1} i_{n-1} a\right] \stackrel{\pi_{n-1}^{\prime}}{\underset{R}{\rightleftharpoons}} w_{n-1}, \quad \text { and } \varphi\left(\pi_{n-1}^{\prime}\right)=i_{n-1} \pi_{n-1}
$$

(iii) $w_{n-1} \ldots w_{1} w_{0}=v_{0}$ and $i_{0} \pi_{0} i_{1} \pi_{1} \ldots i_{n-1} \pi_{n-1}=\rho_{0}$.

It follows that in $P^{\prime}$ there exists a production

$$
p^{\prime} \cdot[A i \alpha] \rightarrow H_{i_{n-1}} \xi\left(\left[X_{n-1} i_{n-1} a\right] \ldots\left[X_{0} i_{0} X_{0}\right]\left[A i \alpha X_{0}\right]\right)
$$

and a derivation $[A i \alpha] \underset{\boldsymbol{R}}{\stackrel{\pi^{\prime}}{\Rightarrow}} w$, such that

(a) $w=a v_{0} v_{1}, \pi^{\prime}=p^{\prime} \rho_{1}^{\prime} \pi_{0}^{\prime} \ldots \pi_{n-1}^{\prime} q^{\prime}$, with $q^{\prime}$ is $H_{i_{n-1}} \rightarrow a$.

(b) $\varphi\left(p^{\prime} \rho_{1}^{\prime}\right)=i \rho_{1}$,

$$
\begin{gathered}
\varphi\left(\pi_{0}^{\prime} \ldots \pi_{n-1}^{\prime}\right)=i_{0} \pi_{0} i_{1} \ldots i_{n-2} \pi_{n-2}, \\
\varphi\left(\pi_{n-1}^{\prime} q^{\prime}\right)=i_{n-1} \pi_{n-1}, \quad \text { and } i_{0} \pi_{0} i_{1} \ldots i_{n-2} \pi_{n-2} i_{n-1} \pi_{n-1}=\rho_{0} .
\end{gathered}
$$

Hence, $\varphi\left(\pi^{\prime}\right)=i \rho_{1} \rho_{0}=i \pi$. 
Now it is not difficult to verify that $G^{\prime}[\bar{r} / \bar{r}] G$. We leave the details to the reader and we only mention that if $[S] \underset{R^{\prime}}{\stackrel{\pi^{\prime}}{\Rightarrow}} w$ then one should distinguish the first production from the remainder of the derivation. A similar argument can be used for the reverse direction (Condition (ii) of the cover definition). This concludes the proof of Lemma 2.2 .

Next we show that any almost GNF grammar can be transformed to a GNF grammar. This is done in the following algorithm. The newly obtained grammar will right cover the original grammar.

Algorithm 2.3. Input. An almost $G N F$ grammar $G=(N, \Sigma, P, S)$. Output. $A G N F$ grammar $G^{\prime}=\left(N^{\prime}, \Sigma, P^{\prime}, S\right)$ such that $G^{\prime}[\bar{r} / \bar{r}] G$. Method. We use two auxiliary sets, $N_{0}$ and $P_{0}$. Initially set $N^{\prime}=N, N_{0}=\emptyset$ and

$$
P_{0}=\{A \rightarrow \alpha\langle i\rangle \mid i \cdot A \rightarrow \alpha \text { is in } P \text { and } \alpha \in \Sigma\} .
$$

Step 1: For each production $i \cdot A \rightarrow B C \alpha$ in $P$ (with $B, C \in N$ and $\alpha \in N^{*}$ ) the following is done.

(i) If $j \cdot C \rightarrow D \beta E$ is in $P$ (with $D, E \in N$ and $\beta \in N^{*}$ ) then, for any pair of productions $k \cdot B \rightarrow a$ and $l \cdot D \rightarrow b$ in $P$ add

and

$$
A \rightarrow a H_{k l} \beta[E j] \alpha\langle i\rangle
$$

$$
H_{k l} \rightarrow b\langle k l\rangle
$$

to $P_{0}$. Add $[E j]$ to $N_{0}$ and $[E j]$ and $H_{k l}$ to $N^{\prime}$.

(ii) If $j \cdot C \rightarrow b$ is in $P$, then, for any production $k \cdot E \rightarrow a$ add

$$
A \rightarrow a H_{k j} \alpha\langle i\rangle
$$

and

$$
H_{k j} \rightarrow b\langle k j\rangle
$$

to $P_{0}$. Add $H_{k j}$ to $N^{\prime}$.

Step 2: Set $P^{\prime}=P_{0}$. For each $[E j]$ in $N_{0}$ add $[E j] \rightarrow \alpha\langle i j\rangle$ to $P^{\prime}$ for each production $E \rightarrow \alpha\langle i\rangle$ in $P_{0}$.

Step 3: Remove the useless symbols.

The general idea of the transformation is displayed in Fig. 2.

Lemma 2.3. Any almost $G N F$ grammar $G$ can be transformed to a $G N F$ grammar $G^{\prime}$ such that $G^{\prime}[\bar{r} / \bar{r}] G$.

Proof. Let $\psi: \Delta_{G^{\prime}} \rightarrow \Delta_{G}^{*}$ be the cover homomorphism which is defined in the algorithm. As we did in the proof of Lemma 2.2 we will use homomorphism $\varphi$ instead of $\psi$. Two claims are used in the proof of Lemma 2.3. For any triple of strings $\alpha, \beta$ and $\gamma$ with $\alpha=\beta \gamma$ we have that $\alpha / 3$ denotes $\gamma$.

Claim 1. Assume $A \in N$. 

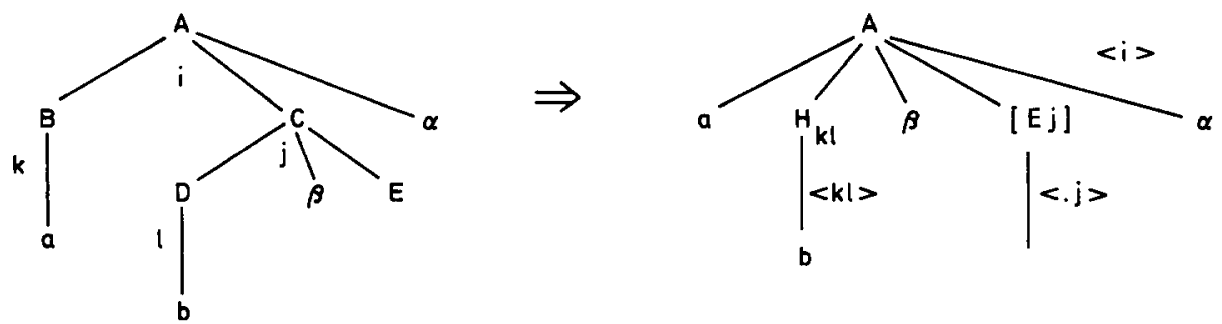

Fig. 2. Step 1 of Algorithm 2.3

(i) If $A \underset{R}{\stackrel{\pi^{\prime}}{\Rightarrow}} w$ in $G^{\prime}$ then $A \stackrel{\varphi\left(\pi^{\prime}\right)}{\longrightarrow} w$ in $G$.

(ii) If $[A k] \underset{R}{\stackrel{\pi^{\prime}}{\Rightarrow}} w$ in $G^{\prime}$ then $A \underset{R}{\stackrel{\delta}{\Rightarrow}} w$ in $G$, with $\delta=\varphi\left(\pi^{\prime}\right) / k$.

Proof of Claim 1. The proof is by induction on $\left|\pi^{\prime}\right|$.

Basis. If $\left|\pi^{\prime}\right|=1$ then we have

(i) Production $A \rightarrow w$ is both in $P$ and $P^{\prime}$, hence the claim is trivially satisfied.

(ii) Production $\pi^{\prime} \cdot[A k] \rightarrow w$ is in $P^{\prime}$. From step 2 of the algorithm it follows that $\varphi\left(\pi^{\prime}\right)=k i$, where $i \cdot A \rightarrow w$ is in $P$. Therefore $A \stackrel{\delta}{\Rightarrow} w$ in $G$, with $\delta=\varphi\left(\pi^{\prime}\right) / k$.

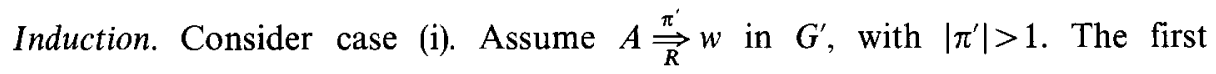
production which is used in this derivation is either of the form $i^{\prime} \cdot A \rightarrow a H_{k l} \beta[E j] \alpha\langle i\rangle$ or $i^{\prime} \cdot A \rightarrow a H_{k j} \alpha\langle i\rangle$. Notice that in both cases we can completely determine from which two productions of $P$ such a production has been constructed. We continue with the former case. The case in which $A \rightarrow a H_{k j} \alpha$ is the first production can be treated similarly and is therefore omitted. Now we can factorize the derivation in the following way:

(a) $i^{\prime} \cdot A \rightarrow a H_{k l} \beta[E j] \alpha$, with $\varphi\left(i^{\prime}\right)=i \cdot A \rightarrow B C \alpha$, where $B$ is the lefthand side of production $k$ in $P$ and $C$ is the lefthand side of production $j$ in $P$.

(b) $\alpha \underset{\boldsymbol{R}}{\stackrel{\pi_{0}^{\prime}}{\longrightarrow}} w_{0}$, and from the induction hypothesis it follows that $\alpha \underset{\vec{R}}{\stackrel{\pi_{0}}{\longrightarrow}} w_{0}$ in $G$, where $\pi_{0}=\varphi\left(\pi_{0}^{\prime}\right)$.

(c) $[E j] \underset{R}{\stackrel{\pi_{1}^{\prime}}{\longrightarrow}} w_{1}$, and from the induction hypothesis it follows that $E \underset{R}{\stackrel{\pi_{1}}{\longrightarrow}} w_{1}$ in $G$, where $\pi_{1}=\varphi\left(\pi_{1}^{\prime}\right) / j$.

(d) $\beta \underset{R}{\stackrel{\pi_{2}^{\prime}}{\longrightarrow}} w_{2}$, and from the induction hypothesis it follows that $\beta \underset{\boldsymbol{R}}{\stackrel{\pi_{2}^{\prime}}{\longrightarrow}} w_{2}$ in $G$, where $\pi_{2}=\varphi\left(\pi_{2}^{\prime}\right)$.

(e) $q^{\prime} \cdot H_{k l} \rightarrow b$, where we assume that $b \in \Sigma$ is the righthand side of production $l$ in $P$. Moreover, $\varphi\left(q^{\prime}\right)=l k$.

It follows that $i^{\prime} \pi_{0}^{\prime} \pi_{1}^{\prime} \pi_{2}^{\prime} q^{\prime}=\pi^{\prime}, a b w_{2} w_{1} w_{0}=w$ and $\varphi\left(\pi^{\prime}\right)=i \pi_{0} j \pi_{1} \pi_{2} l k$, such that (if we assume that $D$ is the lefthand side of production $l$ )

$$
\begin{aligned}
& A \underset{\vec{R}}{\stackrel{i}{\longrightarrow}} B C \alpha \underset{\mathbb{R}}{\stackrel{\pi_{0}}{\longrightarrow}} B C w_{0} \underset{\vec{R}}{\stackrel{j}{\longrightarrow}} B D \beta E w_{0} \underset{R}{\stackrel{\pi_{1}}{\longrightarrow}} B D \beta w_{1} w_{0} \ldots \\
& \ldots \stackrel{\pi_{2}}{\underset{R}{\longrightarrow}} B D w_{2} w_{1} w_{0} \stackrel{l}{\underset{R}{l}} B b w_{2} w_{1} w_{0} \stackrel{k}{\underset{R}{\longrightarrow}} a b w_{2} w_{1} w_{0}=w .
\end{aligned}
$$


This concludes the verification of case (i). Case (ii) can be verified along similar lines and therefore this case is omitted. This concludes the induction part of the proof and therefore the claim is proved.

Claim 2. Consider CFG $G^{\prime}$ before step 3 of the algorithm is executed. If $A \stackrel{\pi}{\vec{R}} w$ in $G$ then there exists $\pi^{\prime} \in \Delta_{G^{\prime}}^{*}$ such that $A \underset{R}{\stackrel{\pi^{\prime}}{\Rightarrow}} w$ in $G^{\prime}$ and $\varphi\left(\pi^{\prime}\right)=\pi$.

Proof of Claim 2. In the proof which may proceed by induction on $|\pi|$ one should distinguish that $A \underset{R}{\stackrel{\pi}{\Rightarrow}} w$ in $G$ can also imply $[A k] \underset{R}{\stackrel{\pi^{\prime}}{\Rightarrow}} w$, for some $k \in \Delta_{G}$ and with $\varphi\left(\pi^{\prime}\right) / k=\pi$. We omit the proof since it proceeds along the same lines as the proof of Claim 1 .

From these two claims it is now clear that $G^{\prime}[\bar{r} / \bar{r}] G$.

The next theorem follows from the previous results.

Theorem 2.4. Any E-free CFG $G$ can be transformed to a $C F G G^{\prime}$ in $G N F$ such that $G^{\prime}[\bar{r} / \bar{r}] G$.

Proof. For any $\varepsilon$-free CFG $G$ we can find an $\varepsilon$-free NLR grammar $G_{0}$ (Corollary $2.3)$ such that $G_{0}[\bar{r} / \bar{r}] G$. The single productions of $G_{0}$ can be eliminated in such a way that the right cover is preserved (Theorem 2.2) and the new grammar, which is also non-left-recursive (Observation 2.3) can be transformed with Algorithm 2.2 followed by Algorithm 2.3 to a grammar $G^{\prime}$ which is in GNF and which has the property $G^{\prime}[\bar{r} / \bar{r}] G$.

Now that we have seen this positive cover result one can ask for analogous results for left covers and left-to-right covers. Unfortunately these results can not be given in such a general way as the right cover result. We will extensively return to this problem in the forthcoming sections. A few positive results on $l / \bar{r}$ and $\bar{r} / l$-covers will be presented here.

Lemma 2.4. Any $C F G G$ in $\overline{G N F}$ can be transformed to a $C F G G^{\prime}$ in $G N F$ such that $G^{\prime}[l / \bar{r}] G$.

Proof. Assume that $G=(N, \Sigma, P, S)$ is in $\overline{\mathrm{GNF}}$. Define $G_{R}=\left(N, \Delta_{G}, P_{R}, S\right)$ with

$$
P_{R}=\{A \rightarrow \alpha i \mid i \cdot A \rightarrow \alpha a \in P, a \in \Sigma\} .
$$

Define a homomorphism $\varphi: \Delta_{G} \rightarrow \Sigma$ by letting $\varphi(i)=a$ if $i \cdot A \rightarrow \alpha a$ is in $P$. Notice that $G_{R}$ is unambiguous. Find for $G_{R}$ an equivalent grammar $G_{L}=\left(N^{\prime}, \Delta_{G}, P_{L}, S^{\prime}\right)$ in GNF (for example, apply to $G_{R}$ a transformation to GNF). Grammar $G^{\prime}$ and the associated cover homomorphism $\psi$ is obtained from $G_{L}$ by defining

$$
P^{\prime}=\left\{i^{\prime} \cdot A^{\prime} \rightarrow a \alpha^{\prime}\langle j\rangle \mid i^{\prime} \cdot A^{\prime} \rightarrow j \alpha^{\prime} \in P_{L} \text { and } \varphi(j)=a\right\} .
$$

We may conclude that $G^{\prime}[l / \bar{r}] G$ if we have verified that $\psi$ is well-defined, that is, if $i^{\prime} \cdot A^{\prime} \rightarrow i \alpha^{\prime}$ and $j^{\prime} \cdot A^{\prime} \rightarrow j \alpha^{\prime}$ are in $P_{L}$, then $i \neq j$ (hence, $i^{\prime} \neq j^{\prime}$ ) implies $\varphi(i) \neq \varphi(j)$.

But this property is trivially satisfied since otherwise we are able to generate sentences of the form $\pi_{1} i \pi_{2}$ and $\pi_{1} j \pi_{2}$ in $L\left(G_{L}\right)$ and since $\varphi\left(\pi_{1} i \pi_{2}\right)=\varphi\left(\pi_{1} j \pi_{2}\right)$ $=w \in L(G)$ we have two different right parses for the same sentence $w$. Since these two right parses are only different in one production this is impossible. 
The usefulness of this lemma will become clear with the following observation. We know (Theorem 2.3 and 'symmetry') that any $\varepsilon$-free NRR grammar $G$ can be transformed to a CFG $G_{0}$ in $\overline{\mathrm{GNF}}$ such that $G_{0}[\bar{r} / \bar{r}] G$. From Lemma 2.4 it follows that we can transform $G_{0}$ to a grammar $G^{\prime}$ in GNF such that $G^{\prime}[l / \bar{r}] G_{0}$ and from transitivity we obtain $G^{\prime}[l / \bar{r}] G$.

Corollary 2.4. Any E-free NRR grammar $G$ can be transformed to a $C F G G^{\prime}$ in GNF such that $G^{\prime}[l / \bar{r}] G$.

A similar result has been obtained in Ukkonen [31 and unpublished].

Once we have a grammar in GNF there is still one more useful transformation which can be applied. The following algorithm is a slight generalization of a method which was first used in [23].

Algorithm 2.4. Input. A CFG $G=(N, \Sigma, P, S)$ in $G N F$. Output. A CFG $G^{\prime}$ $=\left(N^{\prime}, \Sigma, P^{\prime}, S\right)$ in $G N F$ such that $G^{\prime}[\bar{r} / l] G$. Method. Initially set $P^{\prime}$ $=\{A \rightarrow a\langle i\rangle \mid i \cdot A \rightarrow a \in P, a \in \Sigma\}$ and $N^{\prime}=N$. The indexed symbols $H$ which are introduced below are added to $N^{\prime}$.

(i) For each production of the form $i \cdot A \rightarrow a \alpha$ in $P, \alpha \neq \varepsilon$, the following is done. Assume $\alpha=B \gamma, \gamma \in N^{*}$. For any $j_{k} \cdot B \rightarrow b_{k} \gamma_{k}$ in $P, 1 \leqq k \leqq|r h s(B)|$ add

and

$$
A \rightarrow a H_{i j_{k}} \gamma_{k} \gamma\langle\varepsilon\rangle
$$

to $P^{\prime}$.

$$
H_{i j_{k}} \rightarrow b_{k}\left\langle i j_{k}\right\rangle
$$

(ii) Remove all useless symbols.

Theorem 2.5. Any CFG $G$ in $G N F$ can be transformed to a $C F G G^{\prime}$ in $G N F$ such that $G^{\prime}[\bar{r} / l] G$.

Proof. We use two claims to prove the theorem. Homomorphism $\varphi$ is defined as in the proof of Lemma 2.2.

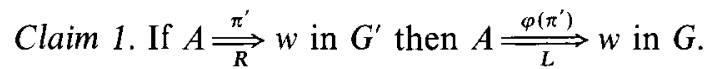

Proof. Notice that $A \in N$. The proof is by induction on $\left|\pi^{\prime}\right|$.

Basis. If $\left|\pi^{\prime}\right|=1$ then $\varphi\left(\pi^{\prime}\right)=\pi^{\prime}$ and the result is clear.

Induction. Assume $\left|\pi^{\prime}\right|=m, m>1$. For $A \underset{\boldsymbol{R}}{\stackrel{\pi^{\prime}}{\longrightarrow}} w$ we may write

$$
A \stackrel{i^{\prime}}{\longrightarrow} a H_{i j_{k}} \gamma_{k} \gamma \stackrel{\stackrel{\rho^{\prime}}{\longrightarrow}}{\longrightarrow} a H_{i j_{k}} w^{\prime} \stackrel{j^{\prime}}{\underset{R}{\longrightarrow}} a b w^{\prime},
$$

where $i^{\prime} \rho^{\prime} j^{\prime}=\pi^{\prime}$ and $a b w^{\prime}=w$.

Since $\left|\rho^{\prime}\right|<m$ and $\gamma_{k} \gamma \in N^{*}$ it is easily verified with the help of the induction hypothesis that $\gamma_{k} \gamma \stackrel{\varphi\left(\rho^{\prime}\right)}{\longrightarrow} w^{\prime}$ in $G$. Moreover, $\varphi\left(i^{\prime}\right)=\varepsilon$ and $\varphi\left(j^{\prime}\right)=j_{k} i$, with $j_{k} \cdot B \rightarrow b \gamma_{k}$ and $i \cdot A \rightarrow a B \gamma$. Hence, $A \stackrel{\varphi\left(\pi^{\prime}\right)}{=} w$ in $G$.

In the following claim we consider grammar $G^{\prime}$ before step (ii) of the algorithm is executed. 
Claim 2. If $A \stackrel{\pi}{\underset{L}{\rightleftharpoons}} w$ in $G$ then there exists $\pi^{\prime} \in \Delta_{\mathbf{G}^{\prime}}^{*}$ such that $A \stackrel{\pi^{\prime}}{\rightleftharpoons} w$ in $G^{\prime}$ and $\varphi\left(\pi^{\prime}\right)=\pi$.

Proof of Claim 2. The argument is similar to that of Claim 1. Notice that if $|\pi|>1$ then we can write

$$
A \underset{L}{\stackrel{i}{\longrightarrow}} a B \gamma \stackrel{j}{\stackrel{j}{\longrightarrow}} a b \gamma_{k} \gamma \stackrel{\rho^{\prime}}{\longrightarrow} a b w^{\prime}=w .
$$

The details are left to the reader.

In both claims we may take $A=S$ and we can conclude that $G^{\prime}[\bar{r} / l] G$.

Theorem 2.5 will be used in the construction of the cover-table.

\section{Counter Example Grammar}

In Ukkonen [33] it is shown that grammar $G$ with productions

$$
\begin{aligned}
& S \rightarrow 0 S L \mid 0 R L \\
& R \rightarrow 1 R L \mid 1 \\
& L \rightarrow \varepsilon
\end{aligned}
$$

can not be left covered with an $\varepsilon$-free CFG. Now consider CFG $G_{0}$ with productions

$$
\begin{aligned}
& \text { 1. } S \rightarrow 0 S L \\
& \text { 2. } S \rightarrow 1 R L \\
& \text { 3. } R \rightarrow 1 R L \\
& \text { 4. } R \rightarrow 2 \\
& \text { 5. } L \rightarrow \varepsilon .
\end{aligned}
$$

Clearly, if $G$ does not have an $\varepsilon$-free CFG which left covers $G$ then also $G_{0}$ does not have such a grammar. Grammar $G_{0}$ will turn out to be useful if we construct the cover table. ${ }^{3}$

In Table 1 we list the productions of a CFG $G_{N}$ which is such that $G_{N}[\bar{r} / l] G_{0}$. Grammar $G_{N}$ is in GNF and since $G_{N}[\bar{r} / l] G_{0}$ we may immediately conclude that $G_{N}$ does not have an $\varepsilon$-free CFG $G^{\prime}$ such that $G^{\prime}[l / \bar{r}] G_{N}$.

We have a few notes on special properties of the grammars $G_{0}$ and $G_{N}$. Grammar $G_{0}$ is both $L L(1)$ and strict deterministic (of degree 1) (cf. Harrison and Havel [9]). Therefore the following result is obvious.

Corollary 3.1. a) Not every $L L(k)$ grammar can be left covered with an $\varepsilon$-free grammar.

b) Not every strict deterministic grammar can be left covered with an $\varepsilon$-free grammar.

3 In Ukkonen [33] not only grammar $G$ but also other counter examples for possible cover results are given 
Table 1. Grammar $G_{N}$

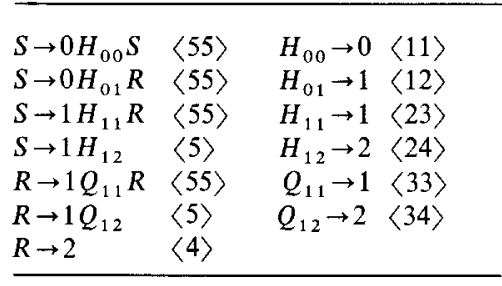

A consequence is that left covering GNF grammars can not be obtained. Notice that for $L L(k)$ grammars this result is in contradiction with excercise 8.1.20 in Aho and Ullman [1].

Both $L L(k)$ and strict deterministic grammars are $L R(k)$ grammars. Therefore the negative results hold for $L R(k)$ grammars as well.

Now we consider grammar $G_{N}$. This grammar is defined in such a way that it is both $L L(2)$ and strict deterministic.

Corollary 3.2. a) Not every $\varepsilon$-free $L L(k)$ grammar can be left-to-right covered with an $\varepsilon$-free grammar.

b) Not every e-free strict deterministic grammar can be left-to-right covered with an e-free grammar.

Also in this case the results hold for $L R(k)$ grammars in GNF as well.

\section{The Cover-Table}

Once more we mention that the context-free grammars which we consider are cycle-free, they do not have useless symbols and if the empty word is in the language then there is exactly one leftmost derivation for this word. Moreover, we will not refer to the special production $S_{0} \rightarrow S \perp$ which may be introduced in the case of elimination of single productions. The cover-table has five rows (ARB, $\varepsilon$-FREE, NLR, $\varepsilon$-FREE NLR, GNF) and seven columns (ARB, $\varepsilon$-FREE, NLR, $\varepsilon$-FREE NLR, GNF, NRR and $\varepsilon$-FREE NRR). Each row has four subrows, one for each type of cover which we consider $(l / l, l / \bar{r}, \bar{r} / l$ and $\bar{r} / \bar{r})$. We use a simple reference system to the entries of the table. Except for the ARB-row all places are labeled with either letters $(a, \ldots, p$. $)$ or numbers $(1 ., \ldots, 96$.$) .$

The details of the construction of the table can be found in the Appendix. Example. Entry 25 . is $n o$. This means that not every $\varepsilon$-free grammar can be left covered with a NLR grammar.

\section{Conclusions}

In the present paper we have given an overview of cover results for some normal forms for context-free grammars. Similar cover results as obtained in this paper 
Table 2. Cover-table

\begin{tabular}{|c|c|c|c|c|c|c|c|c|c|}
\hline$G$ & COVER & $\mathrm{AR}$ & & $\varepsilon$-FREE & NLR & $\begin{array}{l}\varepsilon \text {-FREE } \\
\text { NLR }\end{array}$ & GNF & NRR & $\begin{array}{l}\varepsilon \text {-FREE } \\
\text { NRR }\end{array}$ \\
\hline \multirow[t]{4}{*}{ ARB } & $l / l$ & & yes & yes & yes & yes & yes & yes & yes \\
\hline & $l / \bar{r}$ & & yes & yes & yes & yes & yes & yes & yes \\
\hline & $\bar{r} / l$ & & yes & yes & yes & yes & yes & yes & yes \\
\hline & $\bar{r} / \bar{r}$ & & yes & yes & yes & yes & yes & yes & yes \\
\hline \multirow[t]{4}{*}{ \&-FREE } & $l / l$ & a. & no & 1. yes & 5. no & 9. yes & 13. yes & 17. yes & 21. yes \\
\hline & $l / \bar{r}$ & b. & no & 2. no & 6. no & 10. no & 14. no & 18. yes & 22. yes \\
\hline & $\bar{r} / l$ & & no & 3. no & 7. yes & 11. yes & 15. yes & 19. no & 23. no \\
\hline & $\bar{r} / \bar{r}$ & d. & no & 4. yes & 8. yes & 12. yes & 16. yes & 20. no & 24. yes \\
\hline \multirow[t]{4}{*}{ NLR } & $l / l$ & e. & no & 25. no & 29. yes & 33. yes & 37. yes & 41. no & 45. no \\
\hline & $l / \bar{r}$ & f. & no & 26. yes & 30. yes & 34. yes & 38. yes & 42. no & 46. yes \\
\hline & $\bar{r} / l$ & g. & no & 27. no & 31. yes & 35. yes & 39. yes & 43. no & 47. no \\
\hline & $\bar{r} / \bar{r}$ & h. & no & 28. yes & 32. yes & 36. yes & 40. yes & 44. no & 48. yes \\
\hline$\varepsilon$-FREE & $l / l$ & i. & no & 49. no & 53. no & 57. yes & 61. yes & 65. no & 69. no \\
\hline \multirow[t]{3}{*}{ NLR } & $l / \bar{r}$ & j. & no & 50. no & 54. no & 58. no & 62. no & 66. no & 70. yes \\
\hline & $\bar{r} / l$ & $\mathrm{k}$ & no & 51. no & 55. yes & 59. yes & 63. yes & 67. no & 71. no \\
\hline & $\bar{r} / \bar{r}$ & 1. & no & 52 yes & 56. yes & 60. yes & 64. yes & 68. no & 72. yes \\
\hline \multirow[t]{4}{*}{ GNF } & $l / l$ & $\mathrm{~m}$. & no & 73. no & 77. no & 81. yes & 85. yes & 89. no & 93. no \\
\hline & $l / \bar{r}$ & $\mathrm{n}$. & no & 74. no & 78. no & 82. no & 86. no & 90. no & 94. yes \\
\hline & $\bar{r} / l$ & o. & no & 75. no & 79. yes & 83. yes & 87. yes & 91. no & 95. no \\
\hline & $\vec{r} / \bar{r}$ & $\mathrm{p}$ & no & 76. yes & 80. yes & 84. yes & 88. yes & 92. no & 96. yes \\
\hline
\end{tabular}

will be given in forthcoming papers for regular and deterministically parsable grammars.

The main problems which had to be solved in order to obtain the covertable of Sect. 4 were the elimination of left recursion, the elimination of $\varepsilon$-productions and the problem of finding a right covering grammar in Greibach normal form from an $\varepsilon$-free non-left-recursive grammar. It would be interesting to have a thorough comparison between results for grammar covers and for grammar functors. Unfortunately the elimination of left-recursion does not admit a grammar functor between the original and the non-left-recursive grammar (see e.g. [2]). This does not imply, as has become clear in Hotz $[12,13]$ that the grammar functor approach does not have useful applications when considering normal form transformations.

Acknowledgements. I would like to thank one of the referees for pointing out a few errors in the manuscript and for suggestions concerning the presentation of the results.

\section{References}

1. Aho AV, Ullman JD (1972/1973) The theory of parsing, translation and compiling, Vol. I and II, Prentice Hall, Englewood Cliffs, NJ

2. Benson DB (1977) Some preservation properties of normal form grammars. SIAM J Comput $6: 381-402$ 
3. Cremers AB, Ginsburg S (1975) Context-free grammar forms. J. Comput. System Sci. 11:86-117

4. Foster JM (1968) A syntax improving program. Comput J. 11:31-34

5. Foster JM (1970) Automatic syntactic analysis. MacDonald, London

6. Gray JN, Harrison MA (1969) Single pass precedence analysis, IEEE Conf. Record of the 10th Annual Symposium on Switching and Automata Theory, 106-117

7. Gray JN, Harrison MA (1972) On the covering and reduction problems for context-free grammars. J. Assoc. Comput. Mach. 19:385-395

8. Griffiths TV, Petrick SR (1965) On the relative efficiencies of context-free grammar recognizers. Comm. ACM 8, 289-300

9. Harrison MA, Havel IM (1973) Strict deterministic grammars. J. Comput. System Sci. 7, 237-277

10. Hotz G (1965) Eine Algebraisierung des Syntheseproblems von Schaltkreisen, I und II. Elektron Informationsverarbeit Kybernetik 1:185-231

11. Hotz G (1966) Eindeutigkeit und Mehrdeutigkeit formaler Sprachen. Elektron Informationsverarbeit Kybernetik 2:235-246

12. Hotz G (1978) Normal-form transformations of context-free grammars. Acta Cybernet. 4:65-84

13. Hotz G, Ross RJ (1979) $L L(k)$ - und $L R(k)$-Invarianz von kontextfreien Grammatiken unter einer Transformation auf Greibach Normalform. Elektron Informationsverarbeit Kybernetik 15:73-86

14. Hunt HB, Rosenkrantz DJ (1977) Complexity of grammatical similarity relations. Proc. of the Conf. on Theoretical Computer Science, Waterloo 139-145

15. Hunt HB, Rosenkrantz DJ, Szymanski TG (1976) The covering problem for linear context-free grammars. Theor. Comput. Sci. 2:361-382

16. Hunt HB, Rosenkrantz DJ, Szymanski TG (1976) On the equivalence, containment and covering problems for regular and context-free languages. J. Comput. System Sci. 12:222-268

17. Kuno $S$ (1966) The augmented predictive analyzer for context-free languages - Its relative efficiency. Comm. ACM 9:810-823

18. Kurki-Suonio R (1966) On top-to-bottom recognition and left recursion. Comm. ACM 9:527528

19. Mickunas MD (1976) On the complete covering problem for $L R(k)$ grammars. J. Assoc. Comput. Mach. 23:17-30

20. Mickunas MD, Lancaster RL, Schneider VB (1976) Transforming $L R(k)$ grammars to $L R(1)$, $S L R(1)$ and $(1,1)$ bounded right context grammars. J. Assoc. Comput. Mach. 23:511-533

21. Nijholt A (1979) Grammar functors and covers: From non-left-recursive to Greibach normal form grammars. BIT 19:73-78

22. Nijholt A (1977) On the covering of left-recursive grammars. Conf. Record of the 4th ACM Symposium on Principles of Programming Languages 86-96

23. Nijholt A (1979) From left regular to Greibach normal form grammars. Information Processing Lett. 9:51-55

24. Nijholt A (1979) Structure preserving transformations on non-left-recursive grammars. Proc. 6th Int. Coll. on Automata, Languages and Programming. Maurer HA (ed) Springer, Berlin Heidelberg New York (Lecture Notes in Computer Science Vol. 71 p 446)

25. Nijholt A (1977) On the covering of parsable grammars. J. Comput. System Sci. 15:99-110

26. Nijholt A (1979) A survey of normal form covers for regular grammars. Informatica report 52 , Vrije Universiteit, Amsterdam

27. Paull MC, Unger SH (1968) Structural equivalence of context-free grammars. J. Comput. System Sci. 2:427-463

28. Ross RJ, Hotz G, Benson DB (1978) A general Greibach normal form transformation. CS-78048, Washington State University, Pullman

29. Soisalon-Soininen E (1979) On the covering problem for left-recursive grammars. Theor. Comput. Sci. 8:1-12

30. Stearns RE (1971) Deterministic top-down parsing. Proc. 5th Princeton Conf. on Information Sciences and Systems 182-188

31. Ukkonen E (1978) Transformations to produce certain covering grammars. Proc. 7th Int. Sympos. on Mathematical Foundations of Computer Science. Winkowski J (ed) Springer, Berlin Heidelberg New York (Lecture Notes in Computer Science Vol. 84 p 516)

32. Ukkonen E (1979) Remarks on the non-existence of some covering grammars. Proc. 4th GI Conference on Theoretical Computer Science. Weihrauch K (ed) Springer, Berlin Heidelberg New York (Lecture Notes in Computer Science Vol. 67 p 298) 
33. Ukkonen E (1978) The non-existence of some covering context-free grammars. Information Processing Lett. 8:187-192

34. Wood D (1969) The normal form theorem - another proof. Comput. J. 12:139-147

Received April 19, 1979; revised February 11, 1980

\section{Appendix}

In this Appendix we give the details of the construction of the cover-table (Table 2) of Sect. 4.

A) All the $l / l$ and $\bar{r} / \bar{r}$ entries of the ARB-row are trivially yes. The $l / \bar{r}$ and $\bar{r} / l$ entries are yes because of Theorem 2.1.

B) Trivially yes are also the entries 1., 4., 9., 12., 13., 16., 21., 24., 29., 32., 33., 36., 37. and 40. Because of Theorem 2.1 and Observation 2.2 the entries 30., 31., 34 ., 35., 38. and 39. are yes. Trivially yes are also the entries 57., 60., 61., 64., 85. and 88.

C) Due to grammar $G_{0}$ we have that entry a. is no and from 'symmetry' it follows that entry d. is no. Therefore, also i., $1 ., \mathrm{m}$. and p. are no. Since $G_{0}$ is NLR it follows that entry 5 . is no and again from 'symmetry' entry 20 . is no. Thus, entries 68. and 92. are no.

D) Next we consider grammar $G_{N}$. This grammar has the property that $G_{N}[\bar{r} / l] G_{0}$. Since $G_{0}$ has no $\varepsilon$-free grammar which left covers $G_{0}$ it follows that $G_{N}$ does not have an $\varepsilon$-free grammar which left-to-right covers $G_{N}$. Moreover, $G_{N}$ is in GNF, hence, the entries 14., 10., 6., 2. and b. are all no. Because of 'symmetry' it follows that the entries c., 3., 19. and 23. are no.

We have the following immediate consequences.

(i) Since entries b. and c. are no it follows that entries j., k., n. and o. are no.

(ii) Since entries 2. and 3. are no it follows that entries 50., 51., 74. and 75. are no.

(iii) Since entries 5. and 6. are no it follows that entries 53., 54., 77. and 78. are no.

(iv) Since entries 10. and 14. are no it follows that entries 58., 82., 62. and 86. are no.

(v) Since entries 19. and 23. are no it follows that entries 67., 91., 71. and 95. are no.

E) Due to the Corollaries 2.1 and 2.3 the entries 26., 28. and 52. are yes. From Theorem 2.3 it follows that entry 81 . is yes. From Theorem 2.4 it follows that entries 76., 84. and 96. are yes. Since entry 96. is yes it follows that entries 72. and 48. are yes. From Corollary 2.4 it follows that entry 94 . is yes and, consequently, entries 70., 46. and 22. are yes. Since the entries 81 . and 85 . are yes Theorem 2.5 tells us that entries 83 . and 87 . are yes and, consequently, entries 59., 11., 63. and 15. are yes.

With some simple observations, in which Theorem 2.5 can be used to obtain contradictions, it follows that the entries 73., 90., 93. and 89. are no.

Since the entries 73., 89., 93. and 90. are no we have that entries 49., 69., 65. and 66. are no. Otherwise a contradiction with Theorem 2.3 can be obtained. 
F) Because of Corollary 2.2 we have that entry 8 . is yes and from 'symmetry' it follows that entry 17 . is yes. The assumption that entries $h$. and $g$. are yes leads, with the help of Corollary 2.2, to a contradiction with entries d. and c. are no, respectively. Similarly, with Corollary 2.2 and since entry 31 . is yes, we must conclude that entries $f$. and e. are no in order to avoid contradictions with $h$. and g., respectively.

Since both entry 19. and entry 20 . are no we obtain with the same type of argument that entries 41., 42., 43. and 44. are no. Entry 56. is yes because of entries 8 . and 52. are yes.

The entries 25 . and 27. are both no since otherwise a contradiction can be obtained (via entry 31 . and 56 . in the case of entry 25 . and via entry 56 . in the case of entry 27.) with entry 3 . is no. For any NLR grammar $G$ there exists a NLR grammar $G^{\prime}$ such that $G^{\prime}[\bar{r} / l] G$. Grammar $G^{\prime}$ has an $\varepsilon$-free NLR grammar $G^{\prime \prime}$ such that $G^{\prime \prime}[\bar{r} / l] G$. Hence, entry 55 . is yes and therefore also entry 7 . is yes and ('symmetry') entry 18 . is yes.

Since entries 55. and 56. are yes it follows (with entry 84. is yes) that entries 79. and 80 . are yes.

Both entries 45. and 47. are no because otherwise, with the help of 55. and 56., a contradiction with entry 71 . is no is obtained. This concludes the construction of the cover-table. 